\title{
Paper 174
}

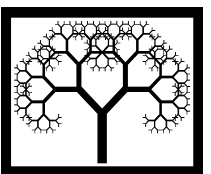

CCivil-Comp Press, 2012

Proceedings of the First International Conference on

Railway Technology: Research, Development and Maintenance,

J. Pombo, (Editor)

Civil-Comp Press, Stirlingshire, Scotland

\section{A Permanent Magnet-Electrically Excited Motor for High-Speed Train Applications}

\author{
M. Schier, F. Rinderknecht and H. Dittus \\ Institute of Vehicle Concepts \\ German Aerospace Center, Stuttgart, Germany
}

\begin{abstract}
The benefits of hub motors are always central to the discussion concerning the choice of the most appropriate drive concept for new high speed trains. This is especially true for low-floor vehicles or double deck vehicles where it is likely that the space, which is now taken by the chassis, will be used as a walkthrough area. The distribution of the drive power between several axles represents a further advantage. In such a concept there is not much space for installing an electric motor close to the wheel in the axial direction of the wheel. In this case it has to be considered if the electric motor could be placed within the wheel. In this paper a special architecture for the design of a wheel hub motor for a high-speed train application is described, the essential concept is shown, and also the advantages and disadvantages of the concept. The result is a concept proposal for an electrically and permanent magnet excited synchronous machine which could be used as a wheel hub motor for a high-speed train application.
\end{abstract}

Keywords: train application, high-speed trains, electrical drive, permanent magnet machines, synchronous machines, permanent magnet-electrical excitation.

\section{Introduction}

Trains with single wheel drives are known since the beginning of the 20th century. The best known example is the so called C-train designed by Ferdinand Porsche, which could drive on the rail and on the road and which could carry heavy loads over long distances or through difficult terrain with curves and mountains. The single wheels of the cars were powered by a generator in the tractor, which was driven by an internal combustion engine. One recognizes the advantageous use of the serial hybrid technology in this case. Nowadays, electric trams with single wheel drives already exist. These drives are designed for speeds of about $80 \mathrm{~km} / \mathrm{h}$. 
The main benefits of distributed propulsion concepts are obvious: they provide a higher drive performance by spreading the force on several axes, resulting in higher climbing ability and redundant systems with increased reliability. This is important not only for propulsion, but also for the braking performance. Finally, the electric high-speed railway traffic on the ground is an environmentally friendly alternative to the national air traffic [1]. The German Aerospace Center, especially with its Institute of Vehicle Concepts, is working on coordinating the special research disciplines which are involved in the development of a high-speed double-deck train concept [2]. One of the disciplines is the development of the drive concept [3], supported by the research activities on electric vehicle drives [4] and the energy management [5]. A second focus is the development of light weight structures [6] to reduce the driving resistances of the vehicle.

A double-deck train concept, which is designed to a length of $202 \mathrm{~m}$ and a total mass of 448 tons, has two end cars and ten middle coaches. The end cars have four axes. Each middle coach has two axis, or 4 wheels as it is shown in Figure 1.

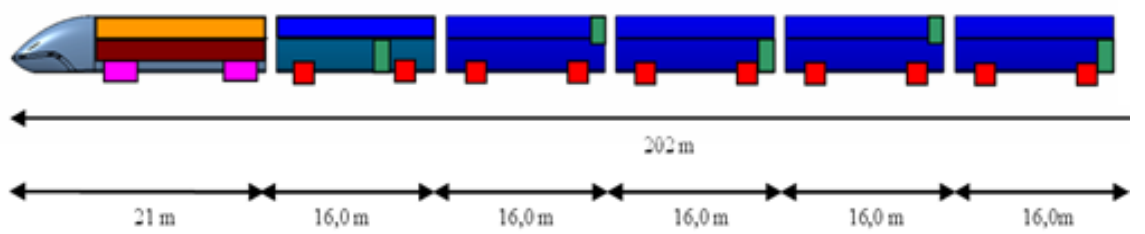

Figure 1: Concept of a double-deck train.

The power to drive a double-deck train with a velocity of $400 \mathrm{~km} / \mathrm{h}$ is nearly up to $18 \mathrm{MW}$, where $8 \mathrm{MW}$ are delivered by the middle coaches. In the given example one wheel has to deliver a power of $250 \mathrm{~kW}$, which is based on a torque of $1.1 \mathrm{kNm}$. The starting torque is greater by a factor of $4(4.4 \mathrm{kNm})$. The motor should have a disk-shaped structure, with an area in the middle of the wheel which is not usable for the electromagnetic parts because of the relatively thick axis, see Figure 2.
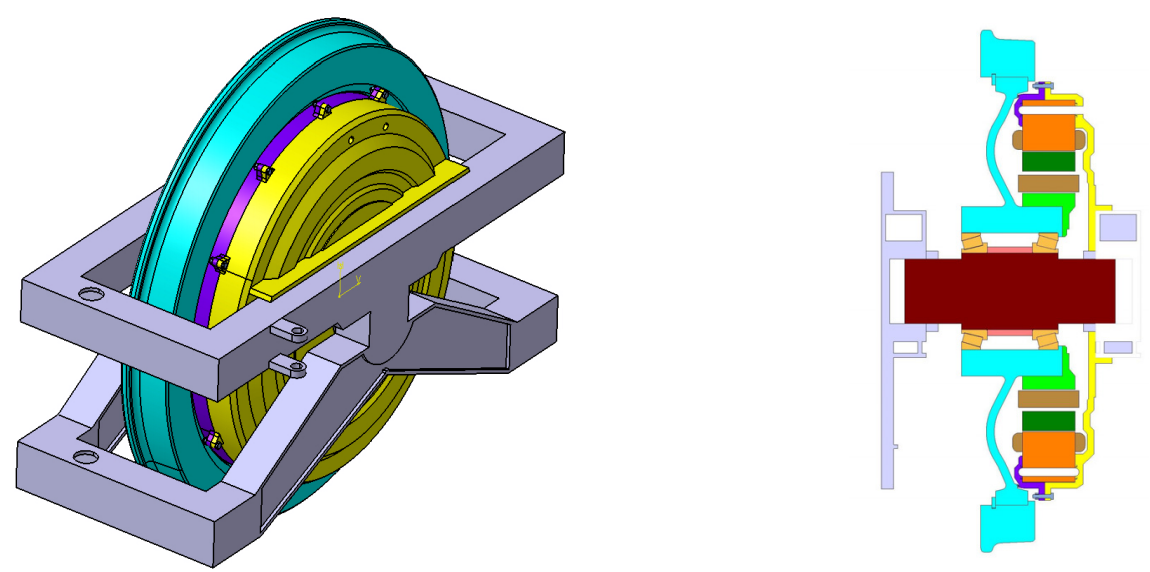

Figure 2: Mechanical boundary conditions and the available space within the wheel to integrate an electric motor. 
These are technically unfavourable conditions compared to the electric drive motors used today. They result in the design of ring-shaped machines with high pole pairs because of the thin iron cores in the radial direction and normally in large winding ends in the axial direction and thus in increased copper costs. It is, therefore, the question how the task can be solved by taking these conditions as an advantage. It should pay attention that the high speeds produce no large magnetic losses due to the ring shaped form of the machine and the high numbers of pole pairs and that the overload rate at the start compared to the driving torque is not too large.

\section{The concept of the electric machine}

\subsection{The basic concept of the electromagnetic architecture}

It has been assumed that the electric machine has a ring shaped form and therefore has a relatively high number of pole pairs. At high speeds this leads to high magnetic losses and high switching losses in the semiconductors of the inverter. When the train rolls out, there should be no losses, either by the magnetization of iron or weakening the field with additional current. Therefore an electrical excitation comes into account. For high numbers of pole pairs, the windings for the electric current required to generate the magnetic potential at the air gap must be applied several times, this is a disadvantage. In addition, the rotor gets heavy. One can reduce the thickness of the iron core in the rotor when the gap flux is generated about half of it by permanent magnets, like it has been investigated to reduce flux leakage in synchronous machines [7]. Then the iron core carries only half of the air gap flux. This saves weight of unneeded iron. Some weight is added by the magnets. It is important to find a compromise here, which allows reducing the weight in comparison to a purely electrically excited machine. The basic design of this parallel circuit of electric and permanent magnetic excitation is shown in Figure 3.
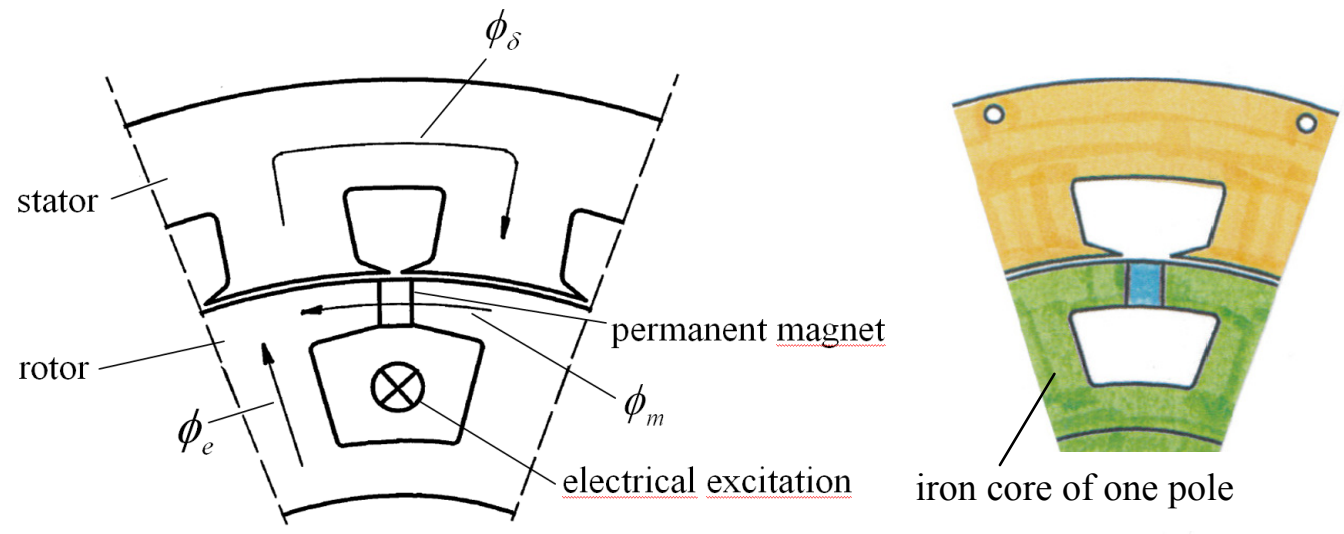

Figure 3: Basic concept of the electromagnetic architecture.

This machine concept is a synchronous machine with a single tooth winding in the stator. Through the use of electrical field windings connected in parallel to the 
magnetic potential of the permanent magnets, the rotor iron cores of each pole no longer carry the total excitation flux of one pole. Ideally, they need to carry only half of the flux, so they are only half as thick. The magnetic potential of the excitation winding, however, remains the same, so that although at a first glance the copper effort is not smaller, but at a second glance, there is more space in the tangential direction for the field winding. This is an advantage for such a ring-shaped machine with its small iron yokes. The magnetic flux of the magnets adds to the flux of the field winding and is thereby generated where it is needed, nearly to the air gap. In the electrically unexcited case, the flux of the permanent magnets closes briefly through the rotor yoke and is therefore magnetically invalid to the outside. The machine is therefore in the electrically-inactive and magnetically-inactive state, which is an automatic safety mechanism to prevent short circuits and blocking in case of error. Moreover, this can result in a very large field weakening area without additional current heat losses, a significant advantage over pure permanent magnet excited machines. Normally the point of the maximum torque or power is not the same point as of the maximum efficiency. The dimensioning of the permanent magnet material is a further issue of the basic investigations. In this case magnets of rare earth material are used. In the following chapter the relations between the magnetic fluxes and the flux densities are described. It follows a comparison between two electromagnetic architectures.

\subsection{Electric Behaviour of the Machine}

\subsubsection{Magnetic Excitation}

Figure 4 shows a simplified magnetic equivalent circuit for the magnetic excitation, neglecting leakage fluxes and without magnetic voltage drops in the iron. The electrical excitation $\theta_{e}$, characterized by its number of windings $N$ and its excitation current $I_{e}$,

$$
\theta_{e}=N \cdot I_{e}
$$

is magnetically connected in parallel to the permanent magnet excitation and defines the magnetic potential $V_{\delta}$ at the air gap and at the magnets.

$$
V_{\delta}=\theta_{e}=H_{C} \cdot d_{m}+\phi_{m} \cdot R_{m}
$$

The permanent magnet is characterized by its coercivity force $H_{C}$ and the magnetic resistance

$$
R_{m}=\mu_{0} \cdot \frac{d_{m}}{A_{m}}
$$

with its thickness $d_{m}$ and its surface $A_{m}$. 
The air gap is the magnetic resistance $R_{\delta}$ of the circuit, depending of the width $\delta$ and the surface of one pole $A_{p}$.

$$
R_{\delta}=\mu_{0} \cdot \frac{\delta}{A_{p}}
$$

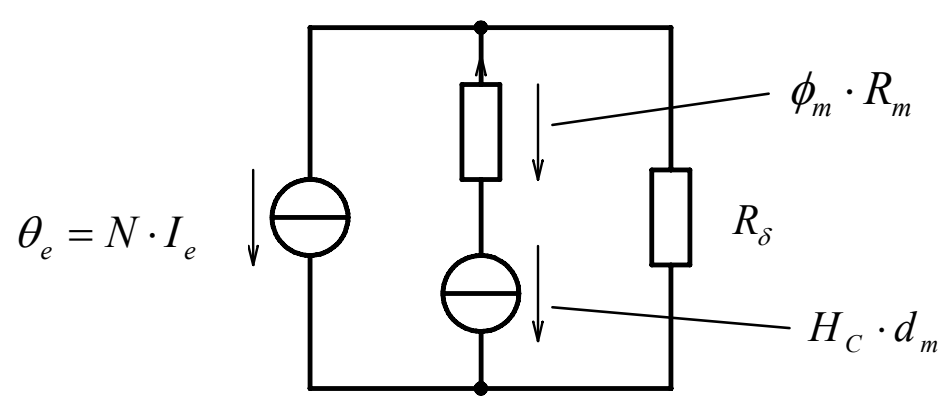

Figure 4: Magnetic equivalent circuit of the combined excitation

Figure 5 shows an example of the flux density in the air gap, in the magnet and in the iron yoke as a function of the excitation current.

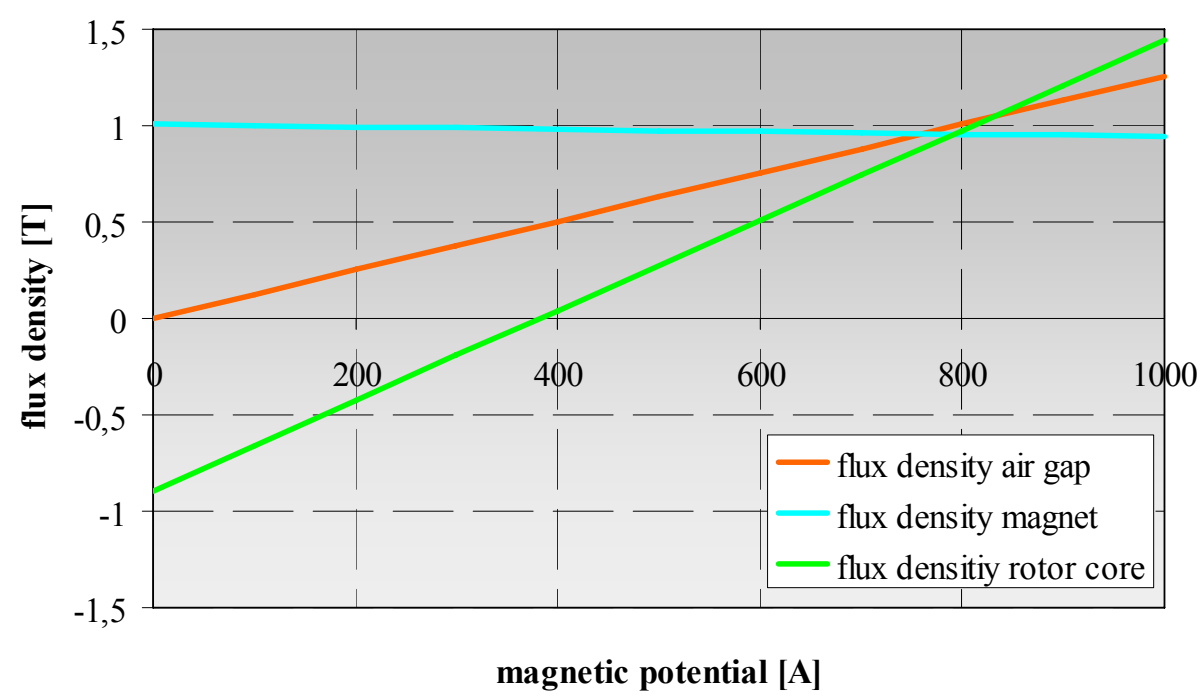

Figure 5: Example of the magnetic flux density in the iron yoke, the magnet and in the air gap in relation to the electrical excitation (magnetic potential)

In the electrically unexcited state, the magnets are magnetically shortcut, their magnetic potential is zero, as well at the air gap, see also Figure 6 on the left side. If the electrical excitation is turned on, the flux through the air gap is half from the magnets and half from the iron core, as it is shown in Figure 6 on the right side. In opposite to purely electrically excited machines the iron core is used with twice of the maximum induction level, each with different polarity. Thus, the magnetically active iron is better utilized. With the electric current the air gap flux density is controlled similar to an electrically excited machine. 


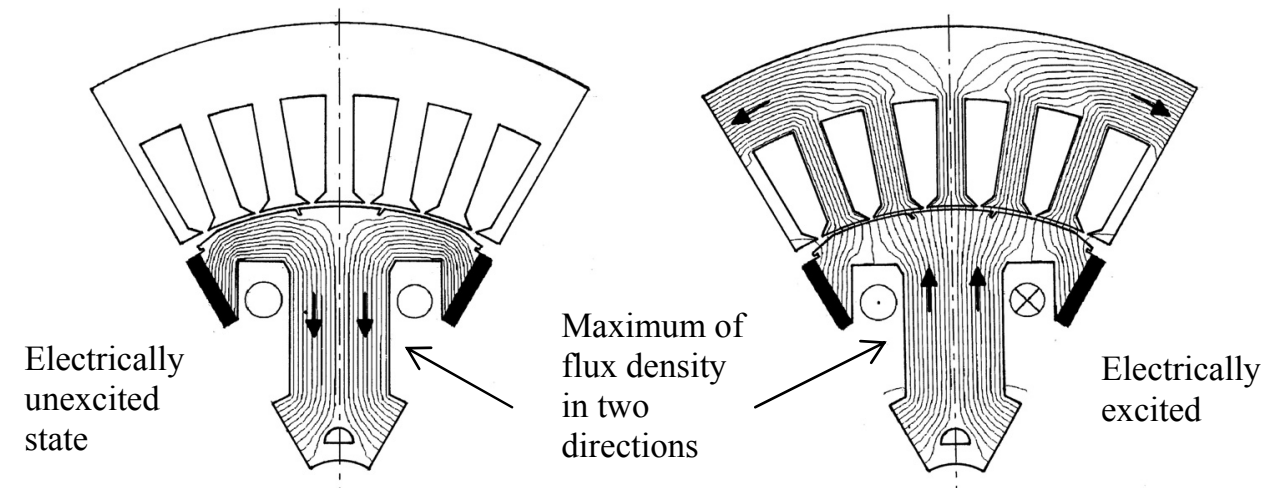

Figure 6: Behaviour of the magnetic field without and with electrical excitation

\subsubsection{Stator Winding}

For the topology of the stator winding two principles are of special concern. These are the combinations of slots to poles from $3 / 2$, using a three-phase machine this results in a number of holes of

$$
q=\frac{N}{2 p \cdot m}=0.5
$$

and the combination 12/10, yielding a number of holes with three phases of 0.4 . Here $N$ is the number of slots, $p$ the number of pole pairs and $m$ the number of phases. Both topologies are shown in Figure 7. In both cases, the coils can either be mounted as movable coils on the stator teeth, or they can be wound into the stator slots. To minimize ripple torque and noise emissions it is advantageous to design the stator slots with a very small opening width as it is shown in the following figure. This increases the costs of the winding in comparison to movable coils. The first topology allows a simple wrapping technique by connecting the following coils on the circumference, the second has a higher winding factor.
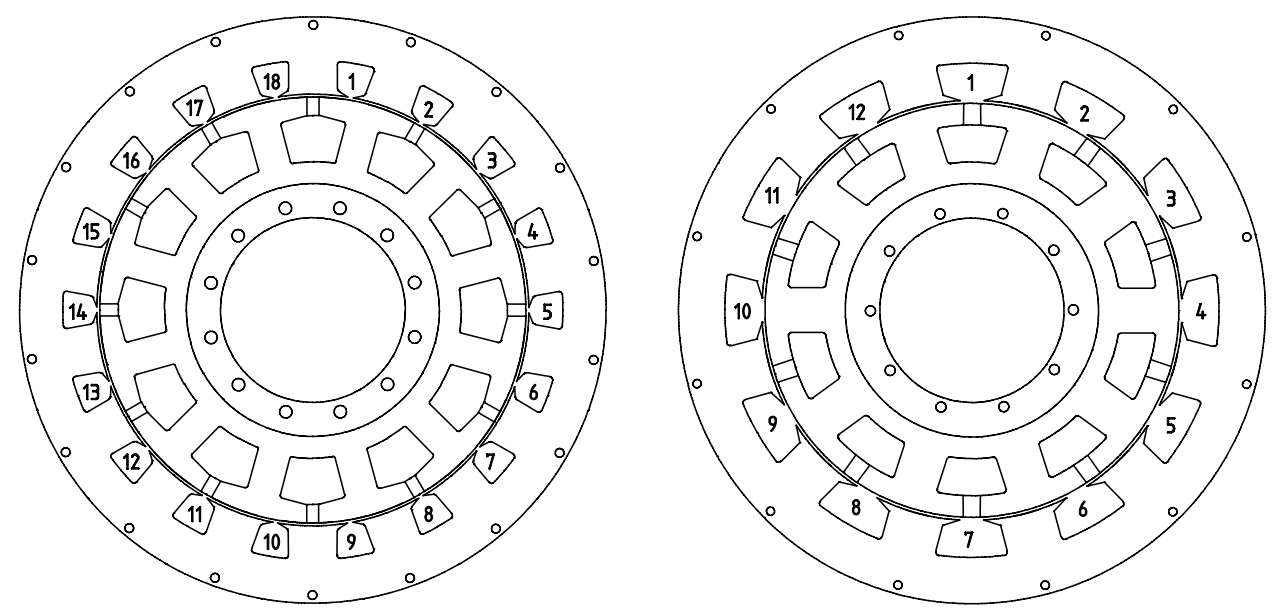

Figure 7: Two electromagnetic topologies of the electromagnetic circuit with $q=0.5$ (left) und $q=0.4$ (right), with numbers of the slots 
The relation of the electromagnetic forces in the slots are shown in Figure 8 . The left image in Figure 8 shows the location of the electromagnetic forces of the first topology with a mechanical angle of $20^{\circ}$ between the $\mathrm{N}=18$ positions of the slots (solid lines) and an electrical angle of $120^{\circ}$ between the electromagnetic forces of the wires in two neighboured slots due to the presence of a common greatest divisor of 6 between the number of slots 18 and the number of pole pairs 6 . Thus, $18 / 6=3$ pointers with a $120^{\circ}$ angle to each other are possible. The electrical angle $\alpha$ between the voltages of two neighboured slots

$$
\alpha=\frac{p}{N} \cdot 360^{\circ}
$$

is $120^{\circ}$. With this topology it is easy to wind the neighboured stator tooth with a coil of the following phase. In one slot the conductors of two coils are positioned. This is shown in the figure by drawing two pointers with the same angle.

For the topology in the right part of Figure 7 there is no common divisor between the number of slots and number of pole pairs. The mechanical spacing of the slots is equal to the electrical angle between two neighboured electromagnetic forces. In this case, this is equal to $360^{\circ} / 12=30^{\circ}$. Here, according to the Equation (5), the electrical angle between the electromagnetic forces in two neighboured slots is equal to $150^{\circ}$.
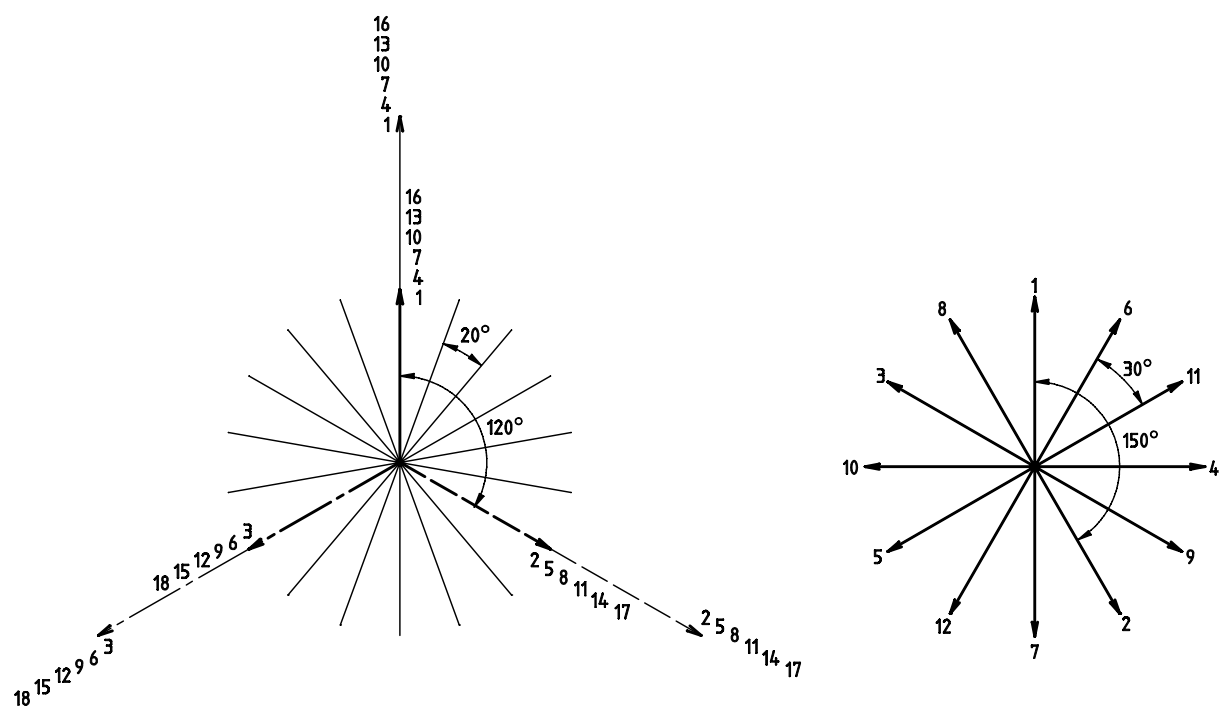

Figure 8: Electromagnetic force of the two electromagnetic topologies with $q=0.5$ (left) and $\mathrm{q}=0.4$ (right)

The wires in the slots with their electromagnetic forces are then connected together to coils, resulting in the induced voltages of the three phases. This is illustrated in Figure 9. It recognizes the different degrees of utilization, which is reflected in the angle between the first voltage and the resultant voltage and results as the winding factor in the power calculation of the machine. 

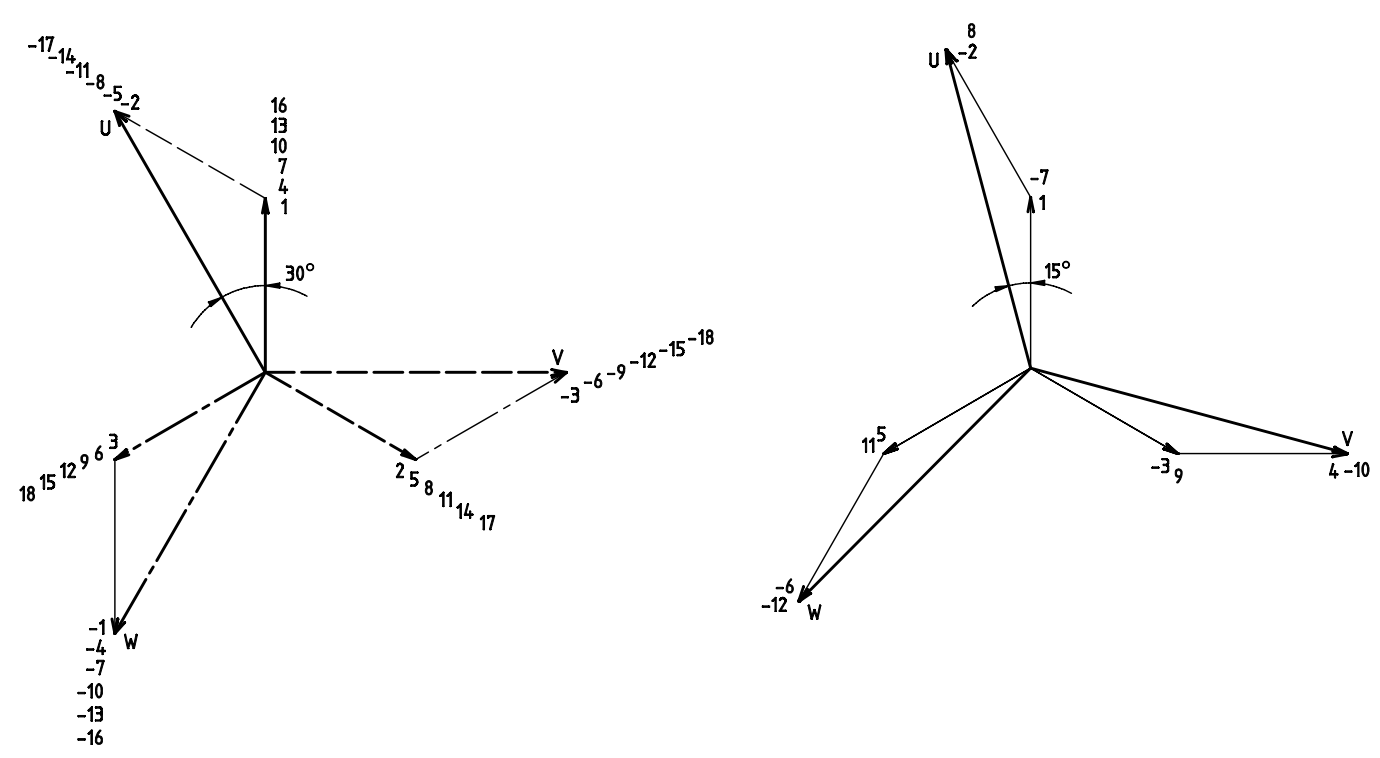

Figure 9: Connecting of the wires in the slots to coils of the two winding topologies, $\mathrm{q}=0.5$ (left, double layer winding), $\mathrm{q}=0.4$ (right, single layer winding)

The winding of the topology in the left part of Figures 7 to 9 has two coil sides lying in a slot (double layer winding), on the right side only one coil side is located in a slot (single layer winding). Both electromagnetic topologies are preferred to be used for the given case.

\subsection{Draft of the mechanical Design}

Figure 10 shows a draft of the wheel hub motor with the given electromagnetic topology. The stationary shaft carries the wheel. The rotor is fixed at the wheel, wherein all rotating parts are made of iron and thus can lead the magnetic flux. Within the rotating system there is no alternating flux. The excitation current can be transmitted to the rotor through slip rings or a high-frequency transformer coupling.

Wheel hub motors in most cases require a special cooling system. They are exposed to the environmental influences directly. The distance between the fixed stator and the rotating parts must be sealed often on a large diameter. The additional power losses of the sealing due to friction are very large. The sealings also are not free of any maintenance. A labyrinth seal protects against the ingress of particles, but not against water under pressure. Due to its large dimensions (large areas) the penetration of moisture or condensation is possible. It is therefore tried to combine the advantages of liquid cooling and the drying effect of air cooling. Therefore, the cooling of the machine partly with liquid, partly with air, is executed. The stator is advantageously cooled with liquid. In addition, cooling air is blown in by a blower which is arranged outside the motor housing. Condensed moisture dries and the end walls, the end windings and the magnets are also cooled, where otherwise the liquid cooling could not reach these parts. 


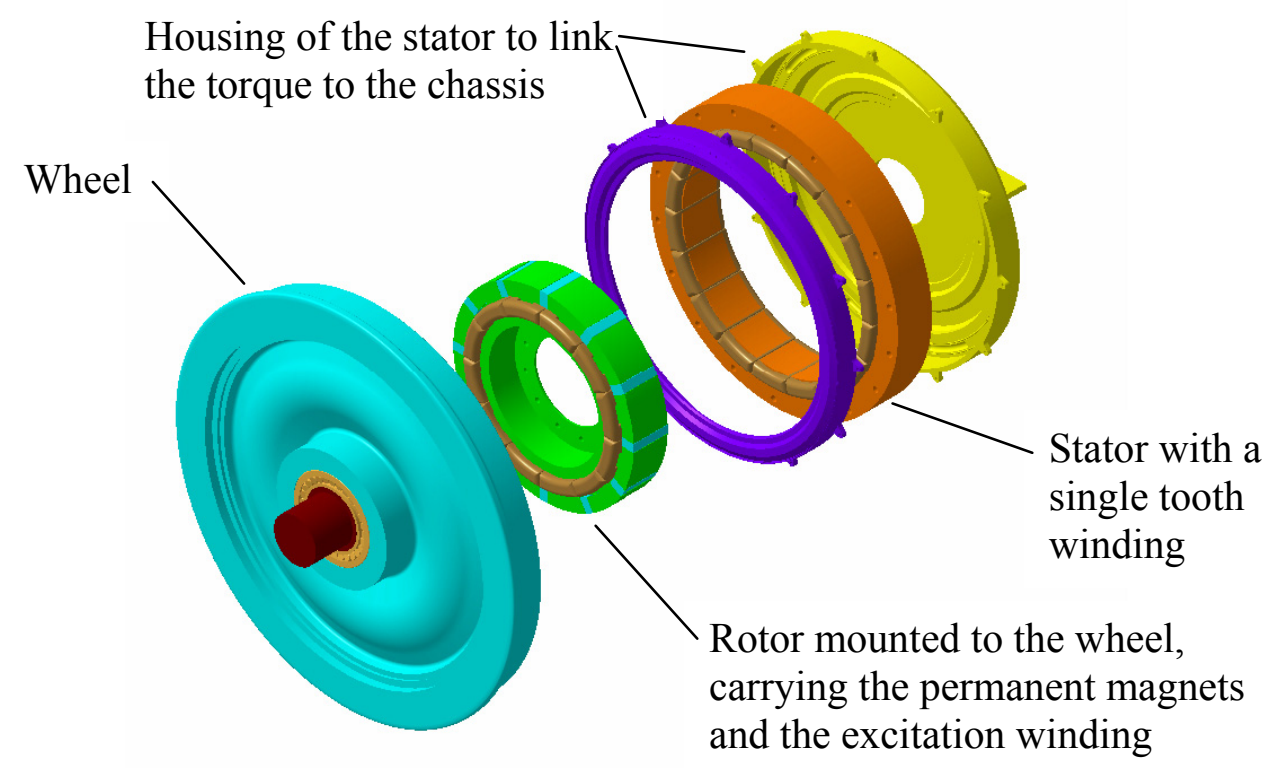

Figure 10: Draft of the wheel hub motor for the high-speed train

\section{Conclusion}

Starting from the question of whether it is possible to transmit the driving power for a high-speed train with single wheel drives to the rail, a concept for a combined electrically-permanent magnet excited synchronous machine is designed. The machine fits into the disk-shaped space of a wheel. It can be controlled by the excitation current from zero to maximum excitation, which is an advantage for the generation of high flux densities during startup, but also in field weakening at high speeds. As a result of the complete de-excitation, the train may roll out without additional losses, which would not be possible in pure permanent magnet excited machines without the use of an additional mechanical coupling. The draft of the machine is based on conceptual designs. The first implementation will be based on hardware samples at a reduced scale.

\section{References}

[1] S. Ehrenberger, M. Fischer, "Study on the potential usage of high speed trains", Europe Rail Technology Review Special, pages 61-65, TZ-Verlag \& Print GmbH, ISBN 978-3-7771-0435-5, ISSN 1869-7801, 2011

[2] G. Kopp, J. König, J. Winter, M. Fischer, S. Ehrenberger, „Systematic derivtion of the NGT rail vehicle concept", Europe Rail Technology Review Special, pages 6-12, TZ-Verlag \& Print GmbH, ISBN 978-3-7771-0435-5, ISSN 1869-7801, 2011 
[3] T. Weiler, "The NGT propulsion concept", Europe Rail Technology Review Special, pages 16-17, TZ-Verlag \& Print GmbH, ISBN 978-3-7771-0435-5, ISSN 1869-7801, 2011

[4] M. Schier, F. Rinderknecht, A. Brinner, H. Hellstern, „High integrated Electric Machine for Aircraft Autonomous Taxiing“, International Conference on Electric Vehicles and Renewable Energies (EVER 11), Monte Carlo, 2011

[5] H. Dittus, J. Winter, "Energy management in rail vehicles", Europe Rail Technology Review Special, pages 37-40, TZ-Verlag \& Print GmbH, ISBN 978-3-7771-0435-5, ISSN 1869-7801, 2011

[6] H. Dittus, J. König. H. E. Friedrich, "Energy reduction of railway vehicles by light weight design", $11^{\text {th }}$ Stuttgart International Symposium, Vol. 2, pp. $501-$ 519, ATZlive Vieweg+Teubner Verlag, Wiesbaden, 2011

[7] W. Freise, "Stator excited Synchronous Machine - Problems of Excitation and Leakage", International Conference on Synchronous Machines, pages 938941, Zürich 1991 